\title{
MEAN VALUE OF MIXED EXPONENTIAL SUMS
}

\author{
HUANING LIU
}

(Communicated by Wen-Ching Winnie Li)

Abstract. For integers $q, m, n, k$ with $q, k \geq 1$, and Dirichlet character $\chi \bmod q$, we define a mixed exponential sum

$$
C(m, n, k, \chi ; q):=\sum_{a=1}^{q} \chi(a) \mathrm{e}\left(\frac{m a^{k}+n a}{q}\right),
$$

where $\mathrm{e}(y)=\mathrm{e}^{2 \pi i y}$, and $\sum_{a}^{\prime}$ denotes the summation over all $a$ with $(a, q)=1$. The main purpose of this paper is to study the mean value of

$$
\sum_{\chi \bmod } \sum_{m=1}^{q}|C(m, n, k, \chi ; q)|^{4},
$$

and to give a related identity on the mean value of the general Kloosterman sum

$$
K(m, n, \chi ; q):=\sum_{a=1}^{q} \chi(a) \mathrm{e}\left(\frac{m a+n \bar{a}}{q}\right),
$$

where $a \bar{a} \equiv 1 \bmod q$

\section{INTRODUCTION}

For integers $q, m, n, k$ with $q, k \geq 1$, and Dirichlet character $\chi \bmod q$, we define a mixed exponential sum

$$
C(m, n, k, \chi ; q):=\sum_{a=1}^{q} \chi(a) \mathrm{e}\left(\frac{m a^{k}+n a}{q}\right),
$$

where $\mathrm{e}(y)=\mathrm{e}^{2 \pi i y}$, and $\sum_{a}^{\prime}$ denotes the summation over all $a$ with $(a, q)=1$.

If $q=p$ is a prime, it follows from A. Weil's work [12] that for all $\chi \bmod p$, and $p \nmid m$,

$$
|C(m, n, k, \chi ; p)| \leq k p^{\frac{1}{2}} .
$$

Let $q=p^{\alpha}$. Using the method established in [3] and [4], T. Cochrane and Z. Zheng [5] obtained the following bound for $C\left(m, n, k, \chi ; p^{\alpha}\right)$.

Received by the editors July 26, 2006 and, in revised form, January 9, 2007.

2000 Mathematics Subject Classification. Primary 11L03, 11L05.

Key words and phrases. Exponential sum, Kloosterman sum, mean value, identity.

This work was supported by the National Natural Science Foundation of China under Grant No.60472068 and No.10671155; Natural Science Foundation of Shaanxi province of China under Grant No.2006A04; and the Natural Science Foundation of the Education Department of Shaanxi Province of China under Grant No.06JK168. 
Proposition 1.1. Let $k \geq 2$ and $\alpha \geq 1$, and let $m, n$ be any integers with $p \nmid m$. If $p>2$, then for all $\chi \bmod p^{\alpha}$, we have $\left|C\left(m, n, k, \chi ; p^{\alpha}\right)\right| \leq k p^{\frac{2}{3} \alpha}\left(n, p^{\alpha}\right)^{\frac{1}{3}}$. If $p=2$, then for all $\chi \bmod 2^{\alpha}$, we have $\left|C\left(m, n, k, \chi ; 2^{\alpha}\right)\right| \leq 2 k 2^{\frac{2}{3} \alpha}\left(n, 2^{\alpha}\right)^{\frac{1}{3}}$.

Moreover, for $\alpha \geq 2$ and $k \geq 2$, T. Cochrane and Z. Zheng [6] proved various upper bounds for $C\left(m, n, k, \chi ; p^{\alpha}\right)$. Specifically, it was shown that if $\chi$ is primitive or $(p, m, n)=1$, then one has $\left|C\left(m, n, k, \chi ; p^{\alpha}\right)\right| \leq 2 k p^{\frac{2}{3} \alpha}$. If $\chi$ has conductor $p$ and $(p, m, n)=1$, then the stronger bound $\left|C\left(m, n, k, \chi ; p^{\alpha}\right)\right| \leq k p^{\frac{2}{3} \alpha}$ was given.

Furthermore, define

$$
\begin{aligned}
& S:=\sum_{a=1}^{p^{\alpha}} \chi(g(a)) \text { e }\left(\frac{f(a)}{p^{\alpha}}\right) ; \quad S(\chi, h):=\sum_{a=1}^{p-1} \chi(a) \mathrm{e}\left(\frac{h(a)}{p}\right) ; \\
& S(\Psi, f ; \chi, g):=\sum_{a \in \mathbb{F}_{q} \backslash \mathcal{S}} \chi(g(a)) \Psi(f(a)),
\end{aligned}
$$

where $\alpha \geq 2, f$ and $g$ are rational functions with integer coefficients, $h$ is a Laurent polynomial, and $\Psi$ is a non-trivial additive character on a finite field $\mathbb{F}_{q}$ of characteristic $p$. T. Cochrane (partly with other coauthors) obtained upper bounds on the above mixed exponential sums step by step in [7]-[10].

It may be interesting to study the mean value of mixed exponential sums. The main purpose of this paper is to study the mean value of

$$
\sum_{\chi \bmod } \sum_{q=1}^{q}|C(m, n, k, \chi ; q)|^{4}
$$

and give the following identity.

Theorem 1.1. Let $q, k$ be positive integers with $(k, q)=1$. Then for any integer $n$ with $(n, q)=1$, we have

$$
\begin{aligned}
& \sum_{\chi \bmod } \sum_{q} \sum_{m=1}^{q}|C(m, n, k, \chi ; q)|^{4} \\
=q^{2} \phi^{2}(q) & \prod_{\substack{p^{\alpha} \| q \\
\alpha \geq 2}}\left(\alpha+1-\frac{\alpha+1+2(k, p-1)}{p}\right) \\
& \times \prod_{p \| q}\left(2-\frac{2((k, p-1)+1)}{p}-\frac{1}{p^{2}}+\frac{(k, p-1)^{2}}{p(p-1)}\right),
\end{aligned}
$$

where $\phi(q)$ is the Euler function, and $\prod_{p^{\alpha} \| q}$ denotes the product over all prime divisors $p$ of $q$ with $p^{\alpha} \mid q$ and $p^{\alpha+1} \nmid q$.

We immediately get the following two corollaries.

Corollary 1.1. Let $q$ be a square-full number and $k$ a positive integer with $(k, q)=$ 1. Then for any integer $n$ with $(n, q)=1$, we have

$$
\sum_{\chi \bmod } \sum_{q}^{q}|C(m, n, k, \chi ; q)|^{4}=q^{2} \phi^{2}(q) \prod_{p^{\alpha} \| q}\left(\alpha+1-\frac{\alpha+1+2(k, p-1)}{p}\right) .
$$


Corollary 1.2. Let $q$ be a square-free number and $k$ a positive integer with $(k, q)=$ 1. Then for any integer $n$ with $(n, q)=1$, we have

$$
\begin{aligned}
& \sum_{\chi \bmod } \sum_{q}^{q}|C(m, n, k, \chi ; q)|^{4} \\
& \quad=q^{2} \phi^{2}(q) \prod_{p \mid q}\left(2-\frac{2((k, p-1)+1)}{p}-\frac{1}{p^{2}}+\frac{(k, p-1)^{2}}{p(p-1)}\right) .
\end{aligned}
$$

Moreover, from Theorem 1.1 we can give some identities on the mean value of general Kloosterman sums. For arbitrary integers $m$ and $n$, the general Kloosterman sum $K(m, n, \chi ; q)$ is defined by

$$
K(m, n, \chi ; q):=\sum_{a=1}^{q} \chi(a) \mathrm{e}\left(\frac{m a+n \bar{a}}{q}\right),
$$

where $a \bar{a} \equiv 1 \bmod q$. For $q=p$ a prime, S. Chowla [2] and A. V. Malyshev [11] proved an upper bound for $K(m, n, \chi ; p)$. For general integer $q>2$, and fixed integer $n$ with $(n, q)=1, \mathrm{~W}$. Zhang [13] showed the identity

$$
\begin{aligned}
& \sum_{\chi \bmod } \sum_{q}^{q}|K(m, n, \chi ; q)|^{4} \\
& \quad=\phi^{2}(q) q^{2} d(q) \prod_{p^{\alpha} \| q}\left(1-\frac{2}{\alpha+1} \cdot \frac{p^{\alpha-1}-1}{p^{\alpha}(p-1)}+\frac{\alpha-4 p^{\alpha-1}}{(\alpha+1) p^{\alpha}}\right),
\end{aligned}
$$

where $d(q)$ is the divisor function.

Now taking $k \equiv-1(\bmod \phi(q))$ in Theorem 1.1 , we have the following:

Corollary 1.3. Let $q$ be a positive integer and $n$ an integer with $(n, q)=1$. Then we have

$$
\begin{aligned}
& \sum_{\chi \bmod } \sum_{q}^{q}|K(m, n, \chi ; q)|^{4} \\
& \quad=q^{2} \phi^{2}(q) \prod_{\substack{p^{\alpha} \| q \\
\alpha \geq 2}}\left(\alpha+1-\frac{\alpha+3}{p}\right) \prod_{p \| q}\left(2-\frac{4}{p}-\frac{1}{p^{2}}+\frac{1}{p(p-1)}\right) .
\end{aligned}
$$

\section{Some LEMmaS}

To prove Theorem 1.1 and Corollary 1.3, we need the following lemmas.

Lemma 2.1. Let $k, q_{1}, q_{2}$ be positive integers with $\left(q_{1}, q_{2}\right)=1$. Then

$$
\left|C\left(m, n, k, \chi ; q_{1} q_{2}\right)\right|=\left|C\left(m q_{2}^{k-1}, n, k, \chi_{1} ; q_{1}\right)\right| \cdot\left|C\left(m q_{1}^{k-1}, n, k, \chi_{2} ; q_{2}\right)\right|
$$

where $\chi=\chi_{1} \chi_{2} \bmod q_{1} q_{2}$ with $\chi_{1} \bmod q_{1}$ and $\chi_{2} \bmod q_{2}$. 
Proof. From the properties of reduced residue systems we have

$$
\begin{aligned}
& C\left(m, n, k, \chi ; q_{1} q_{2}\right)=\sum_{a=1}^{q_{1} q_{2}} \chi(a) \mathrm{e}\left(\frac{m a^{k}+n a}{q_{1} q_{2}}\right) \\
& =\sum_{b=1}^{q_{1}} \sum_{c=1}^{q_{2}} \chi_{1} \chi_{2}\left(b q_{2}+c q_{1}\right) \mathrm{e}\left(\frac{m\left(b q_{2}+c q_{1}\right)^{k}+n\left(b q_{2}+c q_{1}\right)}{q_{1} q_{2}}\right) \\
& =\chi_{1}\left(q_{2}\right) \chi_{2}\left(q_{1}\right) \sum_{b=1}^{q_{1}} \sum_{c=1}^{q_{2}} \chi_{1}^{\prime}(b) \chi_{2}(c) \mathrm{e}\left(\frac{m\left(\left(b q_{2}\right)^{k}+\left(c q_{1}\right)^{k}\right)+n\left(b q_{2}+c q_{1}\right)}{q_{1} q_{2}}\right) \\
& =\chi_{1}\left(q_{2}\right) \chi_{2}\left(q_{1}\right) \sum_{b=1}^{q_{1}} \chi_{1}^{\prime}(b) \mathrm{e}\left(\frac{m q_{2}^{k-1} b^{k}+n b}{q_{1}}\right) \sum_{c=1}^{q_{2}} \chi_{2}(c) \mathrm{e}\left(\frac{m q_{1}^{k-1} c^{k}+n c}{q_{2}}\right) \\
& =\chi_{1}\left(q_{2}\right) \chi_{2}\left(q_{1}\right) C\left(m q_{2}^{k-1}, n, k, \chi_{1} ; q_{1}\right) C\left(m q_{1}^{k-1}, n, k, \chi_{2} ; q_{2}\right) .
\end{aligned}
$$

Therefore,

$$
\left|C\left(m, n, k, \chi ; q_{1} q_{2}\right)\right|=\left|C\left(m q_{2}^{k-1}, n, k, \chi_{1} ; q_{1}\right)\right| \cdot\left|C\left(m q_{1}^{k-1}, n, k, \chi_{2} ; q_{2}\right)\right| .
$$

Lemma 2.2. Let $q, k$ be positive integers and $n_{0}$ an integer with $\left(n_{0}, q\right)=1$. Then

$$
\sum_{m=1}^{q}\left|C\left(m, n_{0}, k, \chi ; q\right)\right|^{4}=\frac{1}{\phi(q)} \sum_{m=1}^{q} \sum_{n=1}^{q}|C(m, n, k, \chi ; q)|^{4} .
$$

Proof. From the properties of reduced residue systems we have

$$
\begin{aligned}
& \phi(q) \sum_{m=1}^{q}\left|C\left(m, n_{0}, k, \chi ; q\right)\right|^{4}=\phi(q) \sum_{m=1}^{q}\left|\sum_{a=1}^{\prime} \chi(a) \mathrm{e}\left(\frac{m a^{k}+n_{0} a}{q}\right)\right|^{4} \\
& =\sum_{m=1}^{q} \sum_{n=1}^{\prime}\left|\sum_{a=1}^{\prime} \chi(a) \mathrm{e}\left(\frac{m a^{k}+n_{0} a}{q}\right)\right|^{4} \\
& =\sum_{m=1}^{q} \sum_{n=1}^{\prime}\left|\sum_{a=1}^{\prime} \chi(a) \mathrm{e}\left(\frac{m \bar{n}^{k} a^{k}+n_{0} a}{q}\right)\right|^{4} \\
& =\sum_{m=1}^{q} \sum_{n=1}^{\prime}\left|\chi(n) \sum_{a=1}^{\prime} \chi(a) \mathrm{e}\left(\frac{m a^{k}+n_{0} n a}{q}\right)\right|^{4} \\
& =\sum_{m=1}^{q} \sum_{n=1}^{\prime}\left|\sum_{a=1}^{q} \chi(a) \mathrm{e}\left(\frac{m a^{k}+n a}{q}\right)\right|^{4} \\
& =\sum_{m=1}^{q} \sum_{n=1}^{q}|C(m, n, k, \chi ; q)|^{4} .
\end{aligned}
$$

Lemma 2.3. Let $p$ be a prime, and let $k$ and $\alpha$ be positive integers. Define

$$
\Psi_{1}\left(k, p^{\alpha}\right):=\sum_{\substack{a=1 \\ p^{\alpha}\left|\left(a^{k}-1\right)\left(b^{k}-1\right) \\ p^{\alpha}\right|(a-1)(b-1)}}^{p^{\alpha}} \sum_{\substack{b=1 \\ p^{\alpha}}}^{\prime} .
$$


Then we have

$$
\Psi_{1}\left(k, p^{\alpha}\right)=(\alpha+1) \phi\left(p^{\alpha}\right)-p^{\alpha-1} .
$$

Proof. Since $p^{\alpha} \mid(a-1)(b-1)$, then $p^{\alpha} \mid\left(a^{k}-1\right)\left(b^{k}-1\right)$. Therefore,

$$
\begin{aligned}
& \Psi_{1}\left(k, p^{\alpha}\right)=\sum_{\substack{a=1 \\
p^{\alpha} \mid(a-1)(b-1)}}^{p^{\alpha}} \sum_{\substack{p^{\alpha} \\
p^{\alpha}}}^{\prime} \\
& =\sum_{\beta=0}^{\alpha} \sum_{\gamma=0}^{\alpha} \sum_{\begin{array}{c}
a=1 \\
\left(a-1, p^{\alpha}\right)=p^{\beta} \\
p^{\alpha} \mid(a-1)(b-1)
\end{array}}^{p^{\alpha}} \sum_{\substack{b=1 \\
\left(a-p^{\alpha}\right)=p^{\gamma}}}^{p^{\alpha}} \\
& =\sum_{\beta=0}^{\alpha} \sum_{\gamma=0}^{\alpha} \sum_{\substack{u=1 \\
\left(u p^{\beta}+1, p\right)=1}}^{p^{\alpha-\beta}} \sum_{\substack{v=1 \\
\left(v p^{\gamma}+1, p\right)=1}}^{p^{\alpha-\gamma}} \\
& p^{\alpha} \mid u v p^{\beta+\gamma} \\
& =\sum_{\substack{\beta=0 \\
\beta+\gamma>\alpha}}^{\alpha} \sum_{\substack{u=1 \\
\left(u p^{\beta}+1, p\right)=1}}^{\alpha} \sum_{\substack{v=1 \\
\left(v p^{\gamma}+1, p\right)=1}}^{p^{\alpha-\beta}}
\end{aligned}
$$

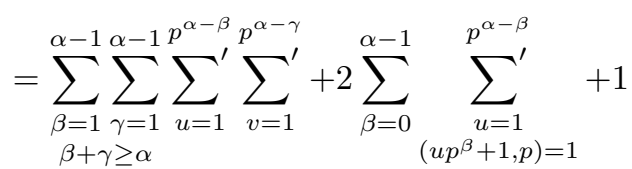

$$
\begin{aligned}
& =\sum_{\beta=1}^{\alpha-1} \phi\left(p^{\alpha-\beta}\right) \sum_{\gamma=\alpha-\beta}^{\alpha-1} \phi\left(p^{\alpha-\gamma}\right)+2\left(\sum_{\substack{u=1 \\
(u+1, p)=1}}^{p^{\alpha}}+\sum_{\beta=1}^{\alpha-1} \sum_{u=1}^{p^{\alpha-\beta}}\right)+1 \\
& =\left((\alpha-1) \phi\left(p^{\alpha}\right)-p^{\alpha-1}+1\right)+2 p^{\alpha-1}(p-2)+2\left(p^{\alpha-1}-1\right)+1 \\
& =(\alpha+1) \phi\left(p^{\alpha}\right)-p^{\alpha-1} \text {. }
\end{aligned}
$$

Lemma 2.4. Let $p$ be a prime, and let $k$ and $\alpha$ be positive integers with $(k, p)=1$. Define

$$
\Psi_{2}\left(k, p^{\alpha}\right):=\sum_{\substack{a=1 \\ p^{\alpha}\left|\left(a^{k}-1\right)\left(b^{k}-1\right) \\ p^{\alpha-1}\right|(a-1)(b-1)}}^{p^{\alpha}} \sum^{p^{\alpha}},
$$

Then we have

$$
\Psi_{2}\left(k, p^{\alpha}\right)= \begin{cases}(\alpha-1+2(k, p-1)) \phi\left(p^{\alpha}\right)-p^{\alpha-1}, & \alpha \geq 2 \\ 2(p-1)(k, p-1)-(k, p-1)^{2}, & \alpha=1\end{cases}
$$


Proof. First we consider the case $\alpha \geq 2$. We have

$$
\begin{aligned}
& \Psi_{2}\left(k, p^{\alpha}\right)=\sum_{\substack{a=1 \\
p^{\alpha}\left|\left(a^{k}-1\right)\left(b^{k}-1\right) \\
p^{\alpha-1}\right|(a-1)(b-1)}}^{p^{\alpha}}=\sum_{\beta=0}^{\alpha} \sum_{\gamma=0}^{\alpha} \sum_{\begin{array}{c}
a=1 \\
\left(a-1, p^{\alpha}\right)=p^{\beta} \\
p^{\alpha} \mid\left(a^{k}-1\right)\left(b^{k}-1\right) \\
p^{\alpha-1} \mid(a-1)(b-1)
\end{array}}^{p^{\alpha}} \sum_{b=1}^{p^{\alpha}} p^{\prime} \\
& =\sum_{\beta=0}^{\alpha} \sum_{\gamma=0}^{\alpha} \quad \sum_{u=1}^{p^{\alpha-\beta}} \quad \sum_{v=1}^{p^{\alpha-\gamma}} \\
& \left(u p^{\beta}+1, p\right)=1\left(v p^{\gamma}+1, p\right)=1 \\
& p^{\alpha} \mid\left(\left(u p^{\beta}+1\right)^{k}-1\right)\left(\left(v p^{\gamma}+1\right)^{k}-1\right) \\
& p^{\alpha-1} \mid u v p^{\beta+\gamma} \\
& =\sum_{\beta=0}^{\alpha} \sum_{\gamma=0}^{\alpha} \sum_{\substack{u=1 \\
\left(u p^{\beta}+1, p\right)=1}}^{p^{\alpha-\beta}} \sum_{\substack{v=1 \\
\left(v p^{\gamma}+1, p\right)=1}}^{p^{\alpha-\gamma}} \\
& p^{\alpha} \mid \sum_{i=1}^{k}\left(\begin{array}{c}
k \\
i
\end{array}\right) u^{i} \sum_{j=1}^{k}\left(\begin{array}{l}
k \\
j
\end{array}\right) v^{j} p^{\beta i+\gamma j} \\
& p^{\alpha-1} \mid p^{\beta+\gamma}
\end{aligned}
$$

Since $p^{\alpha-1} \mid p^{\beta+\gamma}$, then $p^{\alpha} \mid p^{\beta i+\gamma j}$ for $\beta, \gamma \geq 1$ and $i+j>2$. Therefore,

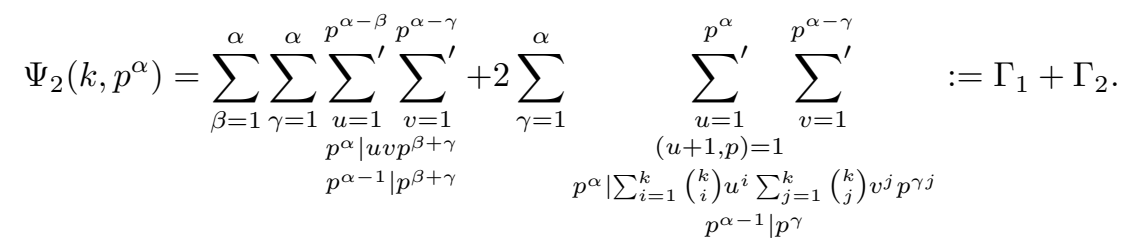

It is not hard to show that

$$
\begin{aligned}
\Gamma_{1} & =\sum_{\substack{\beta=1 \\
\beta+\gamma \geq \alpha}}^{\alpha} \sum_{\substack{\gamma=1 \\
p^{\alpha-\beta}}}^{\prime} \sum_{u=1}^{p^{\alpha-\gamma}} \sum_{v=1}^{\prime}=\sum_{\beta=1}^{\alpha-1} \phi\left(p^{\alpha-\beta}\right) \sum_{\gamma=\alpha-\beta}^{\alpha-1} \phi\left(p^{\alpha-\gamma}\right)+2 \sum_{\beta=1}^{\alpha-1} \sum_{u=1}^{p^{\alpha-\beta}}+1 \\
& =(\alpha-1) \phi\left(p^{\alpha}\right)+p^{\alpha-1} .
\end{aligned}
$$

On the other hand, if $p^{\alpha-1} \mid p^{\gamma}$, then $p^{\alpha} \mid p^{\gamma j}$ for $j \geq 2$. Therefore,

$$
\begin{aligned}
& \Gamma_{2}=2 \sum_{\gamma=1}^{\alpha} \sum_{\begin{array}{c}
u=1 \\
(u+1, p)=1 \\
p^{\alpha} \mid\left((u+1)^{k}-1\right) \sum_{j=1}^{k}\left(\begin{array}{c}
k \\
j
\end{array}\right) v^{j} p^{\gamma j} \\
p^{\alpha-1} \mid p^{\gamma}
\end{array}}^{p^{\alpha}}=2 \sum_{\gamma=1}^{\alpha} \sum_{\begin{array}{c}
u=1 \\
(u+1, p)=1 \\
p^{\alpha} \mid\left((u+1)^{k}-1\right) k v p^{\gamma} \\
p^{\alpha-1} \mid p^{\gamma}
\end{array}}^{p^{\alpha-\gamma}} \sum^{p^{\alpha-\gamma}}
\end{aligned}
$$

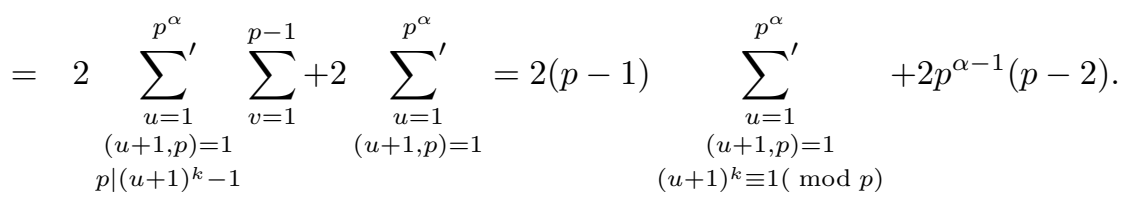


Noting that

$$
\sum_{\substack{u=1 \\(u+1, p)=1 \\(u+1)^{k} \equiv 1(\bmod p)}}^{p^{\alpha}}=p^{\alpha-1} \sum_{\substack{u=1 \\(u+1)^{k} \equiv 1(\bmod p)}}^{p-2}=p^{\alpha-1} \sum_{\substack{u=2 \\ u^{k} \equiv 1(\bmod p)}}^{p-1}=p^{\alpha-1}((k, p-1)-1),
$$

then we have

$$
\Gamma_{2}=2 \phi\left(p^{\alpha}\right)((k, p-1)-1)+2 p^{\alpha-1}(p-2) .
$$

Now combining (2.1), (2.2) and (2.3) we immediately get

$$
\Psi_{2}\left(k, p^{\alpha}\right)=(\alpha-1+2(k, p-1)) \phi\left(p^{\alpha}\right)-p^{\alpha-1}, \quad \text { if } \quad \alpha \geq 2 .
$$

For $\alpha=1$, we easily get

$$
\begin{aligned}
& \Psi_{2}(k, p)=\sum_{\substack{a=1 \\
p \mid\left(a^{k}-1\right)\left(b^{k}-1\right)}}^{p-1} \sum_{\substack{a=1 \\
p \mid a^{k}-1}}^{p-1}+\sum_{\substack{a=1 \\
p \mid b^{k}-1}}^{p-1} \sum_{\substack { b=1 \\
\begin{subarray}{c}{a=1 \\
p\left|a^{k}-1 \\
p\right| b^{k}-1{ b = 1 \\
\begin{subarray} { c } { a = 1 \\
p | a ^ { k } - 1 \\
p | b ^ { k } - 1 } }\end{subarray}}^{p-1} \sum_{b=1}^{p-1} \sum^{p-1} \\
& =2(p-1)(k, p-1)-(k, p-1)^{2} .
\end{aligned}
$$

Lemma 2.5. Let $p$ be a prime, and let $k$ and $\alpha$ be positive integers. Define

$$
\Psi_{3}\left(k, p^{\alpha}\right):=\sum_{\substack{a=1 \\ p^{\alpha-1}\left|\left(a^{k}-1\right)\left(b^{k}-1\right) \\ p^{\alpha}\right|(a-1)(b-1)}}^{p^{\alpha}} \sum_{\substack{p^{\alpha} \\ p^{2}}}^{\prime} \quad \text { and } \Psi_{4}\left(k, p^{\alpha}\right):=\sum_{\substack{a=1 \\ p^{\alpha-1}\left|\left(a^{k}-1\right)\left(b^{k}-1\right) \\ p^{\alpha-1}\right|(a-1)(b-1)}}^{p^{\alpha}} \sum_{\substack{b^{\alpha} \\ p^{2}}}^{\prime} .
$$

Then we have

$$
\Psi_{3}\left(k, p^{\alpha}\right)=(\alpha+1) \phi\left(p^{\alpha}\right)-p^{\alpha-1}
$$

and

$$
\Psi_{4}\left(k, p^{\alpha}\right)= \begin{cases}\alpha \phi\left(p^{\alpha+1}\right)-p^{\alpha}, & \alpha \geq 2 \\ (p-1)^{2}, & \alpha=1\end{cases}
$$

Proof. First we prove (2.4). Since $p^{\alpha} \mid(a-1)(b-1)$, then $p^{\alpha-1} \mid\left(a^{k}-1\right)\left(b^{k}-1\right)$. So from the proof of Lemma 2.3 we easily get

$$
\Psi_{3}\left(k, p^{\alpha}\right)=\sum_{\substack{a=1 \\ p^{\alpha} \mid(a-1)(b-1)}}^{p^{\alpha}} \sum_{\substack{b=1 \\ p^{\alpha}}}^{\prime}=(\alpha+1) \phi\left(p^{\alpha}\right)-p^{\alpha-1} .
$$

Now we prove (2.5). First we suppose that $\alpha \geq 2$. Then from the proof of Lemma 2.3 we have

$$
\Psi_{4}\left(k, p^{\alpha}\right)=p^{2} \sum_{\substack{a=1 \\ p^{\alpha-1}\left|\left(a^{k}-1\right)\left(b^{k}-1\right) \\ p^{\alpha-1}\right|(a-1)(b-1)}}^{p^{\alpha-1} p^{\alpha-1}}=p^{2}\left(\alpha \phi\left(p^{\alpha-1}\right)-p^{\alpha-2}\right)=\alpha \phi\left(p^{\alpha+1}\right)-p^{\alpha} .
$$

For $\alpha=1$, we immediately get

$$
\Psi_{4}(k, p)=\sum_{a=1}^{p-1} \sum_{b=1}^{p-1}=(p-1)^{2} .
$$


Lemma 2.6. Let $p$ be a prime, and let $k$ and $\alpha$ be positive integers with $(k, p)=1$.

Then for any integer $n_{0}$ with $\left(n_{0}, p\right)=1$, we have

$$
\begin{aligned}
& \sum_{\chi \bmod } \sum_{p^{\alpha}}^{p^{\alpha}}\left|C\left(m, n_{0}, k, \chi ; p^{\alpha}\right)\right|^{4} \\
& \quad= \begin{cases}p^{2 \alpha} \phi^{2}\left(p^{\alpha}\right)\left(\alpha+1-\frac{\alpha+1+2(k, p-1)}{p}\right), & \alpha \geq 2 ; \\
p^{2}(p-1)^{2}\left(2-\frac{2((k, p-1)+1)}{p}-\frac{1}{p^{2}}+\frac{(k, p-1)^{2}}{p(p-1)}\right), & \alpha=1 .\end{cases}
\end{aligned}
$$

Proof. By the properties of reduced residue systems we have

$$
\begin{aligned}
\left|C\left(m, n, k, \chi ; p^{\alpha}\right)\right|^{2} & =\sum_{a=1}^{p^{\alpha}} \sum_{b=1}^{p^{\alpha}} \chi(a) \bar{\chi}(b) \mathrm{e}\left(\frac{m\left(a^{k}-b^{k}\right)+n(a-b)}{p^{\alpha}}\right) \\
& =\sum_{a=1}^{p^{\alpha}} \sum_{b=1}^{p^{\alpha}} \chi(a) \mathrm{e}\left(\frac{m b^{k}\left(a^{k}-1\right)+n b(a-1)}{p^{\alpha}}\right) .
\end{aligned}
$$

Then from Lemma 2.2 we get

$$
\begin{aligned}
& \sum_{\chi \bmod } \sum_{p^{\alpha}}^{p^{\alpha}}{ }_{m=1}^{\prime}\left|C\left(m, n_{0}, k, \chi ; p^{\alpha}\right)\right|^{4}=\frac{1}{\phi\left(p^{\alpha}\right)} \sum_{\chi \bmod p^{\alpha}} \sum_{m=1}^{p^{\alpha}} \sum_{n=1}^{p^{\alpha}}\left|C\left(m, n, k, \chi ; p^{\alpha}\right)\right|^{4} \\
& =\frac{1}{\phi\left(p^{\alpha}\right)} \sum_{a=1}^{p^{\alpha}} \sum_{b=1}^{p^{\alpha}} \sum_{c=1}^{p^{\alpha}} \sum_{d=1}^{p^{\alpha}} \sum_{\chi \bmod p^{\alpha}} \chi(a) \bar{\chi}(c) \\
& \times \sum_{m=1}^{p^{\alpha}} \mathrm{e}\left(\frac{m\left(b^{k}\left(a^{k}-1\right)-d^{k}\left(c^{k}-1\right)\right)}{p^{\alpha}}\right) \sum_{n=1}^{p^{\alpha}} \mathrm{e}\left(\frac{n(b(a-1)-d(c-1))}{p^{\alpha}}\right) \\
& =\sum_{a=1}^{p^{\alpha}} \sum_{b=1}^{p^{\alpha}} \sum_{d=1}^{p^{\alpha}} \sum_{m=1}^{p^{\alpha}} \mathrm{e}\left(\frac{m\left(a^{k}-1\right)\left(b^{k}-d^{k}\right)}{p^{\alpha}}\right) \sum_{n=1}^{p^{\alpha}} \mathrm{e}\left(\frac{n(a-1)(b-d)}{p^{\alpha}}\right) \\
& =\sum_{a=1}^{p^{\alpha}} \sum_{b=1}^{p^{\alpha}} \sum_{d=1}^{p^{\alpha}} \sum_{m=1}^{p^{\alpha}} \mathrm{e}\left(\frac{m d^{k}\left(a^{k}-1\right)\left(b^{k}-1\right)}{p^{\alpha}}\right) \sum_{n=1}^{p^{\alpha}} \mathrm{e}\left(\frac{n d(a-1)(b-1)}{p^{\alpha}}\right) \\
& =\sum_{a=1}^{p^{\alpha}} \sum_{b=1}^{p^{\alpha}} \sum_{d=1}^{p^{\alpha}} \sum_{m=1}^{p^{\alpha}} \mathrm{e}\left(\frac{m\left(a^{k}-1\right)\left(b^{k}-1\right)}{p^{\alpha}}\right) \sum_{n=1}^{p^{\alpha}} \mathrm{e}\left(\frac{n(a-1)(b-1)}{p^{\alpha}}\right) \\
& =\phi\left(p^{\alpha}\right) \sum_{a=1}^{p^{\alpha}} \sum_{b=1}^{p^{\alpha}} C_{p^{\alpha}}\left(\left(a^{k}-1\right)\left(b^{k}-1\right)\right) C_{p^{\alpha}}((a-1)(b-1)),
\end{aligned}
$$

where $C_{q}(n)=\sum_{a=1}^{\prime}{ }^{q} \mathrm{e}\left(\frac{a n}{q}\right)$ is the Ramanujan sum. By Theorem 8.6 of [1] we know that $C_{q}(n)=\sum_{d \mid(q, n)} d \mu\left(\frac{q}{d}\right)$, where $\mu$ is the Möbius function. Then for any 
integer $n$,

$$
C_{p^{\alpha}}(n)=\sum_{d \mid\left(p^{\alpha}, n\right)} d \mu\left(\frac{p^{\alpha}}{d}\right)= \begin{cases}\phi\left(p^{\alpha}\right), & \text { if } p^{\alpha} \mid n ; \\ -p^{\alpha-1}, & \text { if } p^{\alpha-1} \| n \\ 0, & \text { if } p^{\alpha-1} \nmid n .\end{cases}
$$

Therefore,

$$
\begin{aligned}
& \sum_{\chi \bmod } \sum_{p^{\alpha}}^{p^{\alpha}}\left|C\left(m, n_{0}, k, \chi ; p^{\alpha}\right)\right|^{4} \\
& =\phi\left(p^{\alpha}\right)\left[\phi^{2}\left(p^{\alpha}\right) \sum_{\substack{a=1 \\
p^{\alpha}\left|\left(a^{k}-1\right)\left(b^{k}-1\right) \\
p^{\alpha}\right|(a-1)(b-1)}}^{p^{\alpha}} \sum_{\substack{b=1 \\
p^{\alpha}}}^{\prime}-p^{\alpha-1} \phi\left(p^{\alpha}\right) \sum_{\substack{a=1 \\
p^{\alpha} \mid\left(a^{k}-1\right)\left(b^{k}-1\right) \\
p^{\alpha-1} \|(a-1)(b-1)}}^{p^{\alpha}}\right. \\
& -p^{\alpha-1} \phi\left(p^{\alpha}\right) \sum_{\substack{a=1 \\
p^{\alpha-1} \|\left(a^{k}-1\right)\left(b^{k}-1\right) \\
p^{\alpha} \mid(a-1)(b-1)}}^{p^{\alpha}}+p^{2 \alpha-2} \sum_{\substack{a=1 \\
p^{\alpha}}}^{p^{\alpha}} \sum_{\substack{b=1 \\
p^{\alpha-1}\left\|\left(a^{k}-1\right)\left(b^{k}-1\right) \\
p^{\alpha-1}\right\|(a-1)(b-1)}}^{p^{\alpha}} \\
& \phi\left(p^{\alpha}\right)\left[p^{2 \alpha} \sum_{\begin{array}{c}
a=1 \\
p^{\alpha} \mid\left(a^{k}-1\right)\left(b^{k}-1\right) \\
p^{\alpha} \mid(a-1)(b-1)
\end{array} \sum^{p^{\alpha}} p^{\alpha}}^{p^{\alpha}}-p^{2 \alpha-1} \sum_{\begin{array}{c}
a=1 \\
p^{\alpha} \mid\left(a^{k}-1\right)\left(b^{k}-1\right) \\
p^{\alpha-1} \mid(a-1)(b-1)
\end{array}}^{p^{\alpha}} \sum_{\substack{b=1 \\
p^{\alpha}}}^{\prime}\right.
\end{aligned}
$$

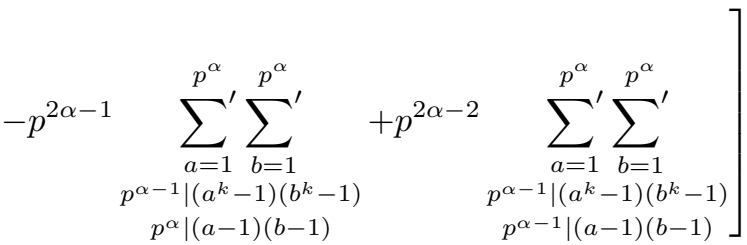

$$
\begin{aligned}
& =p^{2 \alpha} \phi\left(p^{\alpha}\right) \Psi_{1}\left(k, p^{\alpha}\right)-p^{2 \alpha-1} \phi\left(p^{\alpha}\right) \Psi_{2}\left(k, p^{\alpha}\right) \\
& -p^{2 \alpha-1} \phi\left(p^{\alpha}\right) \Psi_{3}\left(k, p^{\alpha}\right)+p^{2 \alpha-2} \phi\left(p^{\alpha}\right) \Psi_{4}\left(k, p^{\alpha}\right) \text {. }
\end{aligned}
$$

Then from Lemma 2.3, Lemma 2.4 and Lemma 2.5 we have

$$
\begin{aligned}
& \sum_{\chi \bmod p^{\alpha}} \sum_{m=1}^{p^{\alpha}}\left|C\left(m, n_{0}, k, \chi ; p^{\alpha}\right)\right|^{4} \\
& \quad= \begin{cases}p^{2 \alpha} \phi^{2}\left(p^{\alpha}\right)\left(\alpha+1-\frac{\alpha+1+2(k, p-1)}{p}\right), & \alpha \geq 2 ; \\
p^{2}(p-1)^{2}\left(2-\frac{2((k, p-1)+1)}{p}-\frac{1}{p^{2}}+\frac{(k, p-1)^{2}}{p(p-1)}\right), & \alpha=1 .\end{cases}
\end{aligned}
$$




\section{Proofs of Theorem 1.1 And Corollary 1.3}

First we prove Theorem 1.1. Let $q=\prod i=1^{r} p_{i}^{\alpha_{i}}, m=\sum_{i=1}^{r} m_{i} \frac{q}{p_{i}^{\alpha_{i}}}$. It is clear that if $m_{i}(i=1,2, \cdots, r)$ runs through a reduced residue system modulo $p_{i}^{\alpha_{i}}$, then $m$ runs through a reduced residue system modulo $q$. So from Lemma 2.1 and Lemma 2.6 we have

$$
\begin{aligned}
& \sum_{\chi \bmod } \sum_{q}^{q}|C(m, n, k, \chi ; q)|^{4} \\
& =\prod_{i=1}^{r}\left[\sum_{\chi \bmod } p_{i}^{\alpha_{i}} \sum_{m_{i}=1}^{p_{i}^{\alpha_{i}}}\left|C\left(m_{i} \frac{q}{p_{i}^{\alpha_{i}}}\left(\frac{q}{p_{i}^{\alpha_{i}}}\right)^{k-1}, n, k, \chi_{i} ; p_{i}^{\alpha_{i}}\right)\right|^{4}\right] \\
& =\prod_{i=1}^{r}\left[\sum_{\chi \bmod p_{i}^{\alpha_{i}}} \sum_{m_{i}=1}^{p_{i}^{\alpha_{i}}}\left|C\left(m_{i}, n, k, \chi_{i} ; p_{i}^{\alpha_{i}}\right)\right|^{4}\right] \\
& =\prod_{\substack{i=1 \\
\alpha_{i} \geq 2}}^{r}\left[\sum_{\chi \bmod p_{i}^{\alpha_{i}}} \sum_{m_{i}=1}^{p_{i}^{\alpha_{i}}}\left|C\left(m_{i}, n, k, \chi_{i} ; p_{i}^{\alpha_{i}}\right)\right|^{4}\right] \\
& \times \prod_{\substack{i=1 \\
\alpha_{i}=1}}^{r}\left[\sum_{\chi \bmod p_{i}^{\alpha_{i}}} \sum_{m_{i}=1}^{p_{i}^{\alpha_{i}}}\left|C\left(m_{i}, n, k, \chi_{i} ; p_{i}^{\alpha_{i}}\right)\right|^{4}\right] \\
& =\prod_{p^{\alpha} \| q}\left[p^{2 \alpha} \phi^{2}\left(p^{\alpha}\right)\left(\alpha+1-\frac{\alpha+1+2(k, p-1)}{p}\right)\right] \\
& \alpha \geq 2 \\
& \times \prod_{p \| q}\left[p^{2}(p-1)^{2}\left(2-\frac{2((k, p-1)+1)}{p}-\frac{1}{p^{2}}+\frac{(k, p-1)^{2}}{p(p-1)}\right)\right] \\
& =q^{2} \phi^{2}(q) \prod_{p^{\alpha} \| q}\left(\alpha+1-\frac{\alpha+1+2(k, p-1)}{p}\right) \\
& \times \prod_{p \| q}\left(2-\frac{2((k, p-1)+1)}{p}-\frac{1}{p^{2}}+\frac{(k, p-1)^{2}}{p(p-1)}\right) .
\end{aligned}
$$

This proves Theorem 1.1.

Now we prove Corollary 1.3. Taking $k \equiv-1(\bmod \phi(q))$, we have

$$
\begin{aligned}
C(m, n, k, \chi ; q) & =\sum_{a=1}^{q} \chi(a) \mathrm{e}\left(\frac{m \bar{a}+n a}{q}\right) \\
& =\sum_{a=1}^{q} \bar{\chi}(a) \mathrm{e}\left(\frac{m a+n \bar{a}}{q}\right)=K(m, n, \bar{\chi}, q) .
\end{aligned}
$$


Noting that $(k, \phi(q))=1$, then from Theorem 1.1 we immediately get

$$
\begin{aligned}
& \sum_{\chi \bmod } \sum_{q}^{q}|K(m, n, \chi ; q)|^{4}=\sum_{\chi \bmod } \sum_{q}^{q}|K(m, n, \bar{\chi} ; q)|^{4} \\
& =\sum_{\chi \bmod } \sum_{q=1}^{q}|C(m, n, k, \chi ; q)|^{4} \\
& =q^{2} \phi^{2}(q) \prod_{\substack{p^{\alpha} \| q \\
\alpha \geq 2}}\left(\alpha+1-\frac{\alpha+3}{p}\right) \prod_{p \| q}\left(2-\frac{4}{p}-\frac{1}{p^{2}}+\frac{1}{p(p-1)}\right) .
\end{aligned}
$$

This completes the proof of Corollary 1.3.

\section{ACKNOWLEDGMENTS}

The author expresses his gratitude to the referee for his detailed comments.

\section{REFERENCES}

[1] T. M. Apostol, Introduction to analytic number theory, Springer-Verlag, New York, 1976. MR0434929 (55:7892)

[2] S. Chowla, On Kloosterman's sum, Norske Vid. Selsk. Forh. (Trondheim), 40 (1967), pp. 7072. MR0228452 (37:4032)

[3] T. Cochrane and Z. Zheng, Pure and mixed exponential sums, Acta Arith., 91 (1999), pp. 249-278. MR1735676 (2000k:11093)

[4] T. Cochrane and Z. Zheng, Exponential sums with rational function entries, Acta Arith., 95 (2000), pp. 67-95. MR1787206 (2001g:11130)

[5] T. Cochrane and Z. Zheng, Bounds for certain exponential sums, Asian J. Math., 4 (2000), pp. 757-773. MR1870657 (2002h:11072)

[6] T. Cochrane and Z. Zheng, Upper bounds on a two-term exponential sum, Sci. China Ser. A, 44 (2001), pp. 1003-1015. MR1857555 (2002g:11125)

[7] T. Cochrane, Exponential sums modulo prime powers, Acta Arith., 101 (2002), pp. 131-149. MR1880304 (2002i:11082)

[8] T. Cochrane and C. Pinner, An improved Mordell type bound for exponential sums, Proc. Amer. Math. Soc., 133 (2005), pp. 313-320. MR2093050 (2005h:11179)

[9] T. Cochrane, J. Coffelt and C. Pinner, A further refinement of Mordell's bound on exponential sums, Acta Arith., 116 (2005), pp. 35-41. MR2114903 (2006b:11100)

[10] T. Cochrane and C. Pinner, Using Stepanov's method for exponential sums involving rational functions, J. Number Theory, 116 (2006), pp. 270-292. MR2195926 (2006j:11113)

[11] A. V. Malyshev, A generalization of Kloosterman sums and their estimates. (Russian), Vestnik Leningrad. Univ., 15 (1960), pp. 59-75. MR0125084 (23:A2391)

[12] A. Weil, On some exponential sums, Proc. Nat. Acad. Sci. U. S. A., 34 (1948), pp. $204-207$. MR0027006 (10:234e)

[13] W. Zhang, On the general Kloosterman sum and its fourth power mean, J. Number Theory, 104 (2004), pp. 156-161. MR2021631 (2005e:11099)

Department of Mathematics, Northwest University, Xi'an, Shaanxi, People's RepubLIC OF CHINA

E-mail address: hnliu@nwu.edu.cn 